\title{
Efecto de la aplicación de Azospirillum sp. y Azotobactersp. sobre el creci- miento y productividad de kikuyo (Pennisetum clandestinum)
}

\author{
Effect of the application of Azospirillum sp. and Azotobactersp. on the growth and produc- \\ tivity of kikuyo (Pennisetum clandestinum)
}

Oscar Vivanco-Galván*, Danny Carrión, Daniel Capa-Mora

DOI. 10.21931/RB/2021.06.04.4

Resumen: El uso de bacterias promotoras de crecimiento vegetal (BPCV), es considerada como una alternativa para sustituir los fertilizantes químicos, ya que favorece la productividad de las especies vegetales. El presente estudio evalúo el efecto de BPCV de los géneros Azospirillum sp. y Azotobacter sp. en el cultivo del Pennisetum clandestinum (kikuyo), sobre altura de la planta, largo máximo de la hoja, largo de raíces, biomasa fresca y proteína total. La aplicación de las bacterias se realizó periódicamente sobre el cultivo, la primera inoculación fue luego del arado del terreno y posterior a ello cada 15 días, hasta el día 45. Los resultados muestran que Azospirillum sp. y Azotobacter sp. influyeron sobre el crecimiento y rendimiento de kikuyo. La aplicación de Azospirillium sp. mostró un incremento significativo en kikuyo sobre los parámetros altura de la planta y el largo de raíz, mientras Azotobacter sp. en la producción de biomasa fresca, largo de raíz y proteína total, por lo cual el uso de estos microorganismos benéficos podría ser de gran importancia en las actividades de producción de pasto para la ganadería, además de ser una alternativa para reducir el uso de productos químicos, con lo que se contribuiría a un mejor manejo de cultivos y al cuidado del medio ambiente.

Palabras clave: Bacterias promotoras de crecimiento vegetal, inoculación bacteriana, crecimiento y productividad de pasto.

\begin{abstract}
The use of plant growth-promoting bacteria (PGPR) is considered an alternative to substitute chemical fertilizers since it favors the productivity of plant species. The present study evaluated the effect of PGPR of the genera Azospirillum sp. and Azotobacter sp. on Pennisetum clandestinum (kikuyo) on plant height, maximum leaf length, root length, fresh biomass, and total protein. The bacteria were applied periodically on the crop, the first inoculation was applied after plowing the soil and every 15 days thereafter, until day 45. The results show that Azospirillum sp. and Azotobacter sp. influenced the growth and yield of kikuyo. The application of Azospirillium sp. showed a significant increase in kikuyo on the parameters plant height and root length, while Azotobacter sp. in the production of fresh biomass, root length, and total protein, so the use of these beneficial organisms could be of great importance in the activities of pasture production for livestock, besides being an aid to reduce the use of chemicals, which would contribute to better crop management and environmental care.
\end{abstract}

Key words: Plant growth promotes bacteria, bacterial inoculation, grass growth, and yield.

\section{Introducción}

La agricultura intensiva depende de una aplicación importante de fertilizantes nitrogenados, junto con otros nutrientes esenciales para maximizar la productividad de los cultivos ${ }^{1}$. Como una alternativa al uso de fertilizantes químicos en la agricultura se ha incrementado el uso de bacterias promotoras del crecimiento vegetal (BPCV) como una efectiva herramienta para mejorar la salud de las plantas ${ }^{2,3}$. Dentro de este grupo importante de BPCV encontramos a especies de Azotobacter y Azospirillum que son ampliamente asociadas con la fijación de nitrógeno ${ }^{4,5}$. Por tal razón estas bacterias también estimulan la producción de hormonas de crecimiento como auxinas, citoquininas y giberelinas, mejorando la absorción de otros nutrientes como el fósforo. Por lo tanto, la inoculación con BPCV es una tecnología promisoria para el aumento de la producción agrícola, mientras se reduce los impactos ambientales por el uso inadecuado de fertilizantes ${ }^{6}$.

El Pennisetum clandestinum (kikuyo) es un hierba de zonas tropicales capaz de crecer mucho en verano y es usado en la alimentación de ganado vacuno. Sin embargo la producción de leche puede verse limitada por la calidad relativamente baja del kikuyo ${ }^{7}$, por esta razón es importante pensar en mejo- rar la calidad del pasto a través de la inoculación de bacterias. Por ejemplo en el caso de Azospirillum la inoculación en hierba forrajera puede minimizar la degradación del suelo y mejorar la producción masiva de forraje. Esto es soportado por un reporte de Boddey et al. ${ }^{8}$ sobre bacterias fijadoras en la rizósfera de las hierbas forrajeras, lo que sugiere un futuro de menor uso de fertilizantes de $\mathrm{N}$ en los pastos forrajeros tropicales ${ }^{8}$. De hecho, la inoculación de la hierba Brachiaria spp. con cepas de Azospirillum brasilense aumentó la producción media de masa de forraje en un $13 \%{ }^{9}$. Asimismo, algunas investigaciones han mostrado que el crecimiento de diferentes plantas, incluidas hierbas anuales y perennes ha incrementado por la aplicación de Azospirillum y Azotobacter. Las bacterias son capaces de incrementar la germinación de las semillas, crecimiento y rendimiento de diferentes cultivos de plantas ${ }^{10-12}$.

Con estos antecedentes y considerando que en Loja existen grandes extensiones de terreno ocupadas por kikuyo usado como alimento del ganado vacuno y como un cultivo importante, el presente trabajo tiene por objetivo evaluar el efecto del Azospirillum sp. y Azotobacter sp. sobre el crecimiento y la productividad del kikuyo. 


\section{Materiales y métodos}

\section{Sitio experimental}

El estudio se realizó en la Estación Agropecuaria-Universidad Técnica Particular de Loja (EA- UTPL) (Ecuador), situada en el sector de Cajanuma, al sur del cantón Loja, a 9 km desde la ciudad de Loja (X: -4,0886 y Y: -79,2066) ${ }^{13}$. El sitio de estudio esta ubicado a una altura de 2.300 m s.n.m., además, presenta medias anuales de temperatura y precipitación de $15,4^{\circ} \mathrm{C}$ y 780 mm, respectivamente, según datos de la Estación Meteorológica "La Argelia"14.

\section{Obtención cepas}

Las dos cepas bacterianas fueron aisladas a partir de suelo de la rizósfera de kikuyo y preservadas a $-80^{\circ} \mathrm{C}$ hasta su posterior uso. Azospirillum sp. y Azotobacter sp. fueron reactivadas y proveídas en cajas petri por la colección de microorganismos de la Universidad Técnica Particular de Loja (UTPL), para Azospirillum sp. en medio Agar Rojo Congo $\left(27^{\circ} \mathrm{C}\right)$, mientras que, en agar Ashby la bacteria Azotobacter sp. $\left(37^{\circ} \mathrm{C}\right)$, ambas permanecieron en incubación y en posición invertida en la obscuridad hasta su uso.

\section{Incremento de biomasa del inoculo bacteriano}

El ensayo se llevó a cabo en el Laboratorio de Cultivo y Conservación de Microorganismos de la UTPL. Para incrementar la biomasa de Azospirillum sp. una colonia de la caja petri fue colocada en medio JMV estéril (20 minutos, $120^{\circ} \mathrm{C}$ y 1.5 atm) sin nitrógeno, luego incubado (Yamato IC802) a $27^{\circ} \mathrm{C}$ entre 48 y 72 horas. En cuanto a Azotobacter sp. una colonia de la caja petri fue inoculada en medio JMV y mantenida a $37^{\circ} \mathrm{C}$ durante 48 y 72 horas. Se realizó una evaluación del número de unidades formadoras de colonias (UFC) de cada cepa bacteriana a través del conteo de bacterias por el método de Neubauer. Una concentración final de $1 \times 10^{8} \mathrm{~g} / \mathrm{mL}$ fue ajustada en cada cepa bacteria en un volumen de $1 \mathrm{~L}$ y mantenida a $4^{\circ} \mathrm{C}$ hasta su posterior aplicación en campo. Una hora aproximadamente transcurrió desde que las botellas de medio fueron transportadas hasta la EA- UTPL.

\section{Diseño experimental}

El experimento fue desarrollado en un área específica de $360 \mathrm{~m}^{2}$, dividido en parcelas de $40 \mathrm{~m}^{2}$ (9 parcelas experimentales), dejando entre parcela una distancia de $4 \mathrm{~m}$, para minimizar efectos de borde. Los tratamientos evaluados fueron: i) Azotobacter sp., ii) Azospirillum sp. y iii) testigo (control, sin aplicación de bacterias). Cada tratamiento tuvo tres repeticiones bajo un diseño experimental de bloques al azar.

\section{Aplicación bacteriana}

Previo a la realización del ensayo un área geográfica con kikuyo y de características homogéneas del terreno fue preparada a través de arado y cruce por método tradicional a través de un tractor (Pascuali Sinea K5 AR) con el propósito de descompactar y airear el suelo para realizar el ensayo.

Para aplicar las bacterias sobre el kikuyo germinado se diluyó $1 \mathrm{~L}$ del concentrado bacteriano en $20 \mathrm{~L}$ de agua, obteniendo una concentración de $1 \times 10^{6}$ células por $\mathrm{mL}$, se usó el proceso mecánico de pulverización ${ }^{15}$. Este procedimiento fue aplicado al kikuyo en varios días. La primera aplicación ocurrió al siguiente día (después de arado), luego a los 15 días (germi- nación) 30 días (crecimiento bajo 10-15 cm), y 45 días (crecimiento medio $25-30 \mathrm{~cm}$ ). El registro de datos se realizó luego de la primera aplicación bacteriana hasta los 60 días, fecha a partir de la cual el pasto está listo para el consumo animal.

\section{Evaluación de variables}

Se evaluaron algunas variables en el kikuyo para determinar el efecto de las cepas bacterianas: i) altura de planta: se colocó una regla graduada perpendicularmente al nivel del suelo y se tomó la altura (cm); ii) largo máximo de la hoja: fue medido una sola vez al final del ensayo (60 días), a partir del peciolo, utilizando la vena central como referencia (cm); iii) largo de raíces: usando una cuadricula de $20 \times 20 \mathrm{~cm}$ se hizo un corte y se obtuvo una muestra que contenía suelo y raíces, se separaron las raíces y se procedió a la obtención de los datos (cm) al final del ensayo; iv) biomasa vegetal fresca: se usó un cuadrante de $1 \mathrm{~m}^{2}$ para cortar y luego pesar en el momento de la cosecha, los datos se reportaron en $\mathrm{kg} / \mathrm{ha}$; v) proteína total a partir de la biomasa cruda: evaluada por el método de Kjeldahl validada por Harris ${ }^{16}$.

\section{Análisis estadísticos}

Para comparar el resultado del efecto de las bacterias sobre las variables evaluadas en $P$. clandestinum se realizó un análisis de varianza de una vía (ANOVAS) $(p<0.05)$. Cuando se determinó significancia, se aplicó la prueba de Tukey subconjuntos homogéneos $(p<0,05)$. Previo al análisis de los datos y una vez calculados los residuos se verificó el cumplimiento de los supuestos de normalidad, independencia y homogeneidad de varianzas. El software estadístico SPSS v. 23.0.17, fue usado para los análisis. Para obtener datos precisos en todas las variables evaluadas se registró 5 mediciones de cada plántula de cada tratamiento al azar, con tres repeticiones.

\section{Resultados y discusión}

La aplicación de las cepas bacterias Azopirillum sp. y Azotobacter sp. en kikuyo, ha permitido evaluar a través de la medición de algunas variables los posibles efectos sobre la productividad y el crecimiento.

El uso de bacterias BPCV en la agricultura esta incrementando continuamente, ya que ofrece una herramienta eficaz para sustituir el uso de fertilizantes químicos, pesticidas y otros suplementos dañinos ${ }^{2,3}$. Azopirillum sp. y Azotobacter sp. son consideradas BPCV por la capacidad de mejorar la salud de las plantas ${ }^{18}$

Los géneros Azospirillium, Azotobacter y Pseudomonas son ampliamente utilizados por sus características como fijadores de nitrógeno ${ }^{19,20}$, la capacidad de producir índoles ${ }^{20,21}$ y solubilizar fósforo ${ }^{22,23}$, propiedades que hacen de estos microorganismos potenciales biofertilizantes.

Se registró la altura de la planta de pasto kikuyo y se determinó que el tratamiento con Azopirillum sp. presenta una mayor altura de la planta (Figura 1) en relación al tratamiento con Azotobacter sp. y al control (testigo). El análisis estadístico realizado evidencia las diferencias significativas entre el tratamiento Azopirillum sp. y el testigo.

Sobre la evaluación de la altura de kikuyo, el tratamiento con Azopirillum sp. mostró los registros de mayor altura, en relación al trabajo de Guimarães et al..$^{24}$ en un estudio con Urochloa brizantha (hierba empalizada) inocularon nueve aislados de Azopirillum y determinaron plantas más altas usando la cepa Azopirillum (AZO2) cuando se comparó con el control. da 
Silva Ramos et al. ${ }^{25}$ evaluó el crecimiento de plantas de maíz inoculadas con Azospirillum lipoferum (BR cepa 11084) luego de 30 días de sembradas observaron un incremento en la altura de la planta.

Sobre el largo máximo de la hoja no se evidencia ningún efecto del uso de Azopirillum sp. y Azotobacter sp. ya que los valores registrados sobre el largo de la hoja son similares y no son estadísticamente diferentes entre tratamientos (Figura 2).

En cuanto al largo máximo de la hoja no de observó una diferencia de la aplicación de Azopirillum sp. y Azotobacter sp. sobre el control. En un estudio realizado por Reddy et $a l^{26}$ en la aplicación de Azopirillum sp. y Azotobacter sp. sobre tomate (Lycopersicon esculentum) donde se evaluó la variación del área de la hoja no se observaron diferencias con el control que no estaba inoculado por ningún microorganismo. Barassi et al. ${ }^{27}$ reportó sobre el mejoramiento en los parámetros fotosintéticos de hojas, incluyendo el incremento en el contenido de clorofila y conductancia estomatal, además, de la producción de biomasa y altura de la planta.

El largo de la raíz que fue medido al día 60 permitió determinar que la presencia de Azopirillum sp. y Azotobacter sp. ejercen un efecto en el desarrollo de la raíz. Los resultados de la aplicación de bacterias muestran una clara diferencia estadística con el testigo (Figura 3).

El incremento en el desarrollo de las raíces se observó en los tratamientos con la aplicación de Azopirillum sp. y Azotobacter sp., debido al mejoramiento de la absorción de agua y nutrientes, lo que ha permitido un incremento de la capacidad

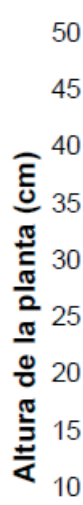

50

45

30

5

0

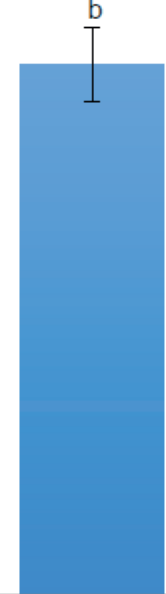

Azospirillum

a

35
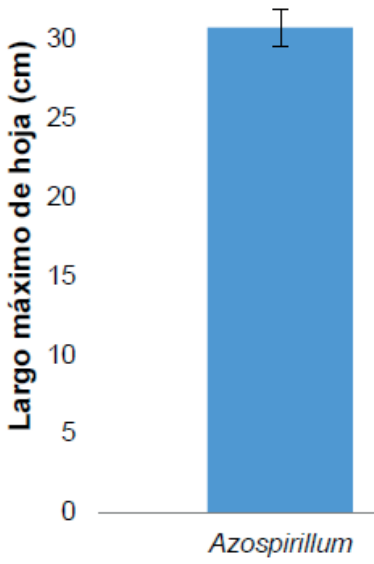

$a b$

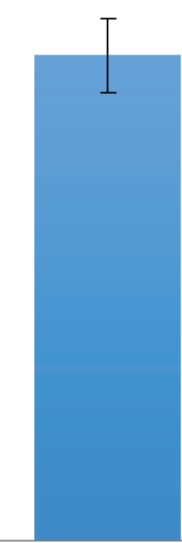

Azotobacter

Tratamientos

a

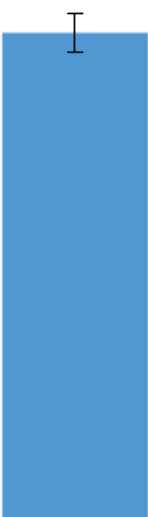

Azotobacter

Tratamientos de la planta para tolerar estrés ambiental como sequía, salinidad, resultando en plantas más vigorosas y productivas ${ }^{28-30}$. En el 2017 Pérez y Sánchez ${ }^{31}$ determinaron que Azopirillum y Azotobacter asociadas al cultivo de Ipomoea batata tuvo incrementos significativos en la longitud de raíz. En Azotobacter se da la producción de la fitohormona auxina que ayuda a la producción de raíces largas e incrementa el número de pelos y raíces laterales que están involucradas en la absorción de nutrientes ${ }^{32}$

La biomasa vegetal fresca de kikuyo se determinó por metro cuadrado, se pudo determinar que el tratamiento Azopirillum sp. presenta un incremento en la biomasa en relación al testigo (Figura 4).

La biomasa vegetal fresca del kikuyo resulto ser mayor en el tratamiento Azotobacter sp. con relación al testigo. En un estudio desarrollado por Mahato y Kafle en el $2018^{33}$ en trigo donde se realizaron varios tratamientos se determinó que la biomasa del tratamiento Azotobacter fue mayor solo respecto al control, además, se observó un alto rendimiento de biomasa en aquellos tratamientos en los que Azotobacter acompañó al fertilizante químico.

La proteína total evaluada en el tratamiento Azotobacter sp. muestra un mayor porcentaje de proteína determinada en relación al tratamiento Azopirillum sp. y testigo (Figura 5).

El porcentaje de proteína determinado en el tratamiento Azotobacter sp. fue mayor en relación a los otros dos tratamientos. En el caso de la inoculación de Azotobacter dentro de condiciones controladas o in vitro, las plantas responden

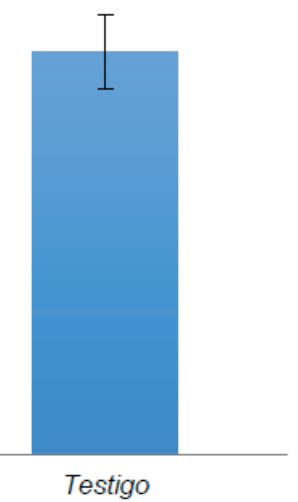

a

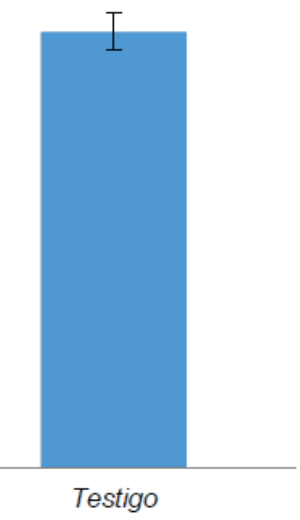

Figura 1. Altura de Pennisetum clandestinum inoculado con tratamiento 1 (Azospirillum sp.), tratamiento 2 (Azotobacter sp.) y tratamiento 3 (Testigo).
Figura 2. Largo máximo de hoja de Pennisetum clandestinum inoculado con tratamiento 1 (Azospirillum sp.), tratamiento 2 (Azotobacter sp.) y tratamiento 3 (Testigo). 


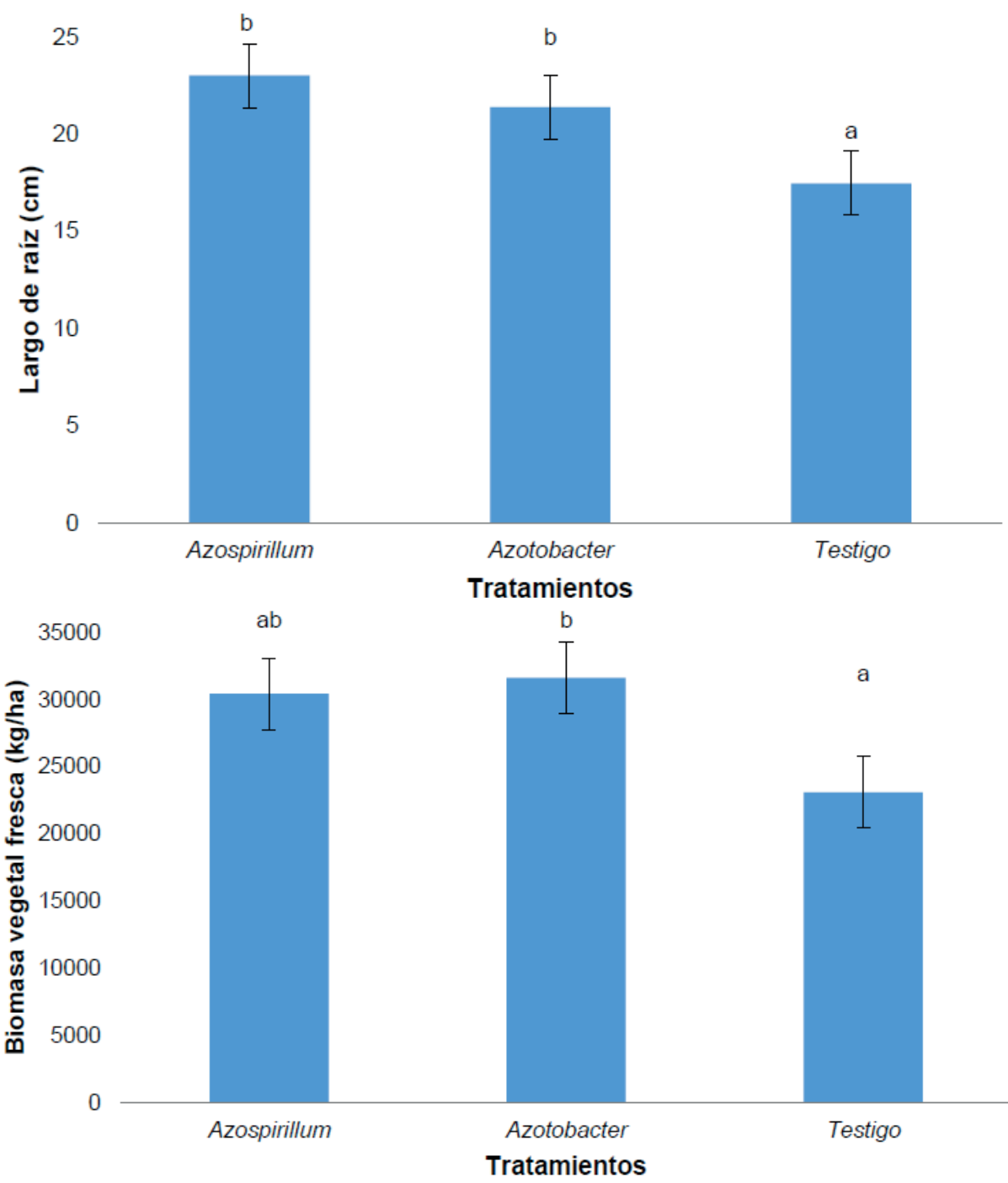

Figura 3. Largo de la raíz de Pennisetum clandestinum inoculado con tratamiento 1 (Azospirillum sp.), tratamiento 2 (Azotobacter sp.) y tratamiento 3 (Testigo).

18

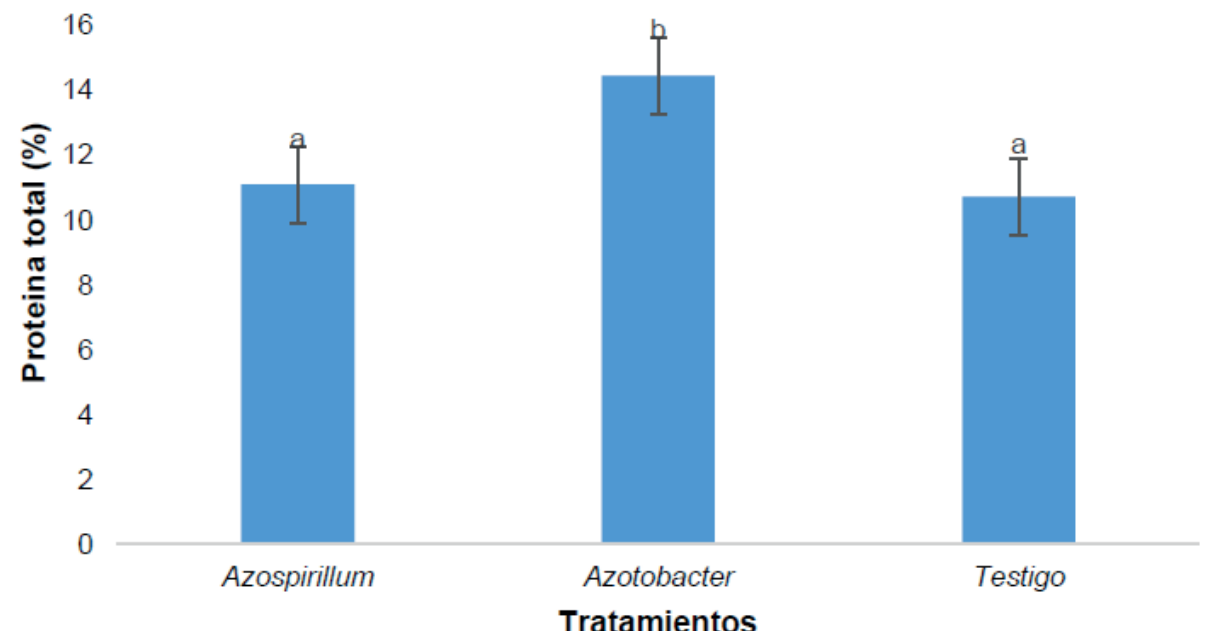

Figura 4. Biomasa vegetal fresca en $\mathrm{Kg} / \mathrm{ha}$ de Pennisetum clandestinum inoculado con tratamiento 1 (Azospirillum sp.), tratamiento 2 (Azotobacter sp.) y tratamiento 3 (Testigo)

Figura 5. Proteína total en porcentaje de Pennisetum clandestinum inoculado con tratamiento 1 (Azospirillum sp.), tratamiento 2 (Azotobacter sp.) y tratamiento 3 (Testigo).

al estímulo bacteriano, entre ellos podemos destacar varios atributos como el contenido de proteína después de haber sido cosechado ${ }^{34}$

Como una perspectiva futura es importante evaluar el efecto de las dos bacterias fijadoras de nitrógeno como parte de un mismo tratamiento ya que se ha reportado en otros estudios que el uso de la especie Azotobacter como biofertilizador o en combinación con otras especies benéficas como

Azospirillum, mejora el rendimiento y la calidad de diferentes cultivos $^{34}$. Asimismo, las bacterias asociadas con la producción de gramíneas que se han estudiado más, son las de los géneros: Azospirillum, Azotobacter, Klebsiella, Beijerinckia, Pseudomonas y Bacillus. Algunas de ellas forman estructuras de resistencia para favorecer su supervivencia en condiciones de estrés, en especial sequía, la cual es común en los pastizales de zonas áridas ${ }^{35}$. 


\section{Conclusiones}

La aplicación de bacterias fijadoras del nitrógeno como Azospirillum sp. y Azotobacter sp. permiten mejorar los indicadores de crecimiento y rendimiento de kikuyo. La bacteria Azotobacter sp. presenta un efecto sobre la producción de biomasa fresca, largo de raíz y proteína total y la bacteria Azospirilum sp. sobre la altura de la planta y el largo de raíz favoreciendo los indicadores de crecimiento y calidad del kikuyo $(P$. clandestinum).

\section{Agradecimientos}

A la Estación Agropecuaria-Universidad Técnica Particular de Loja, por habernos brindado el área para la implementación de los ensayos.

\section{Referencias bibliográficas}

1. Bindraban, P. S., Dimkpa, C., Nagarajan, L., Roy, A., \& Rabbinge, R. (2015). Revisiting fertilisers and fertilisation strategies for improved nutrient uptake by plants. Biology and Fertility of Soils, 51(8), 897-911.

2. Ansari, R. A., Rizvi, R., Sumbul, A., \& Mahmood, I. (2017). PGPR: current vogue in sustainable crop production. In Probiotics and plant health (pp. 455-472). Springer, Singapore.

3. Ansari, R. A., \& Mahmood, I. (Eds.). (2019 a). Plant health under biotic stress: volume 1: organic strategies. Springer.

4. Lakshminarayana, K. (1993). Influence of Azotobacter on nitrogen nutrition of plants and crop productivity. Proceedings of the Indian National Science Academy. Part B Biological sciences, 59(3-4), 303-307.

5. de Souza Moreira, F. M., Da Silva, K., Nóbrega, R. S. A., \& De Carvalho, F. (2010). Bactérias diazotróficas associativas: diversidade, ecologia e potencial de aplicações. Comunicata Scientiae, 1(2), 74-74.

6. Domingues Duarte, C. F., Cecato, U., Trento Biserra, T., Mamédio, D., \& Galbeiro, S. (2020). Azospirillum spp. in grasses and forages. Review. Revista mexicana de ciencias pecuarias, 11(1), 223-240.

7. Fulkerson, B., Griffiths, N., Sinclair, K., \& Beale, P. (2010). Milk production from kikuyu grass-based pastures. Primefacts, 1068, 1-13.

8. Boddey, R. M., Xavier, D. F., Alves, B. J., \& Urquiaga, S. (2003). Brazilian agriculture: the transition to sustainability. Journal of Crop Production, 9(1-2), 593-621.

9. Hungria, M., Nogueira, M. A., \& Araujo, R. S. (2016). Inoculation of Brachiaria spp. with the plant growth-promoting bacterium Azospirillum brasilense: An environment-friendly component in the reclamation of degraded pastures in the tropics. Agriculture, Ecosystems \& Environment, 221, 125-131.

10. Döbereiner, J., \& Day, J. M. (1975). Nitrogen fixation in rhizosphere of grasses. Nitrogen Fixation by Free-Living.

11. Bloemberg, G. V., \& Lugtenberg, B. J. (2001). Molecular basis of plant growth promotion and biocontrol by rhizobacteria. Current opinion in plant biology, 4(4), 343-350.

12. Basak, B. B., \& Biswas, D. R. (2010). Co-inoculation of potassium solubilizing and nitrogen fixing bacteria on solubilization of waste mica and their effect on growth promotion and nutrient acquisition by a forage crop. Biology and Fertility of Soils, 46(6), 641-648.

13. Google. (2020). Mapa de Loja, Ecuador en google maps. Recuperado 5 de marzo de 2020, de https://www.google.com/maps/place/4 '05'18.7\%22S+79¹2'24.2\%22W/@-4.0885363,-79.2078093,18z/ data=!4m21!1m14!4m13!1m6!1m2!1s0x91cb312b7b1dcb03:0x1e 829107f2cfe281!2sEstación+agropecuaria+\%22UTPL\%22!2m2!1 d-79.2066924!2d-4.0884491!1m5!1m1!1s0x91

14. TuTiempo.net. (2017). Estación meteorológica «La Argelia». Recuperado 5 de marzo de 2020 de https://www.tutiempo.net/cli$\mathrm{ma} / 12-2017 /$ ws-8427 00.html

15. Riquelme, J., \& Bustos, C. (2016). Sistema de aplicación innovativo en tomates bajo invernadero. Operación, mantención y calibre del equipo.

16. Harris, L. E. (1970). Métodos para el análisis químico y la evaluación biológica de alimentos para animales (No. SF97 H2).
17. Arbuckle, J. L. (2014). Amos (version 23.0) [computer program]. Chicago: IBM SpSS.

18. Sumbul, A., Ansari, R. A., Rizvi, R., \& Mahmood, I. (2020). Azotobacter: A potential bio-fertilizer for soil and plant health management. Saudi Journal of Biological Sciences, 27(12), 3634.

19. Ahmad, F., Ahmad, l., \& Khan, M. S. (2008). Screening of free-living rhizospheric bacteria for their multiple plant growth promoting activities. Microbiological research, 163(2), 173-181.

20. Yasmin, F., Othman, R., Sijam, K., \& Saad, M. S. (2009). Characterization of beneficial properties of plant growth-promoting rhizobacteria isolated from sweet potato rhizosphere. African Journal of Microbiology Research, 3(11), 815-821.

21. Dawwam, G. E., Elbeltagy, A., Emara, H. M., Abbas, I. H., \& Hassan, M. M. (2013). Beneficial effect of plant growth promoting bacteria isolated from the roots of potato plant. Annals of Agricultural Sciences, 58(2), 195-201.

22. Khan, A. A., Jilani, G., Akhtar, M. S., Naqvi, S. M. S., \& Rasheed, M. (2009). Phosphorus solubilizing bacteria: occurrence, mechanisms and their role in crop production. J agric biol sci, 1(1), 48-58.

23. Sashidhar, B., \& Podile, A. R. (2009). Transgenic expression of glucose dehydrogenase in Azotobacter vinelandii enhances mineral phosphate solubilization and growth of sorghum seedlings. Microbial biotechnology, 2(4), 521-529.

24.Guimarães, S., Bonfim-Silva, E. M., Polizel, A., \& Campos, D. (2011). Produção de capim-marandu inoculado com Azospirillum spp. Enciclopédia Biosfera, 7(13).

25. da Silva Ramos, A., dos Santos, T. M. C., de Santana, T. M., Guedes, E. L. F., \& Montaldo, Y. C. (2010). Ação do Azospirillum lispoferum no desenvolvimento de plantas de milho. Revista Verde de Agroecologia e Desenvolvimento Sustentável, 5(4), 18.

26. Reddy, S., Singh, A. K., Masih, H., Benjamin, J. C., Ojha, S. K., Ramteke, P. W., \& Singla, A. (2018). Effect of Azotobacter sp. and Azospirillum sp. on vegetative growth of Tomato (Lycopersicon esculentum). Journal of Pharmacognosy and Phytochemistry, 7(4), 2130-2137.

27. Barassi, C. A., Sueldo, R. J., Creus, C. M., Carrozzi, L. E., Casanovas, W. M., \& Pereyra, M. A. (2008). Potencialidad de Azospirillum en optimizer el crecimiento vegetal bajo condiciones adversas. Azospirillum sp.: cell physiology, plant interactions and agronomic research in Argentina. Argentina: Asociación Argentina de Microbiologia, 1, 49-59.

28. Bashan, Y., \& Holguin, G. (1997). Azospirillum-plant relationships: environmental and physiological advances (1990-1996). Canadian Journal of Microbiology, 43(2), 103-121.

29. Dobbelaere, S., Croonenborghs, A., Thys, A., Ptacek, D., Vanderleyden, J., Dutto, P., \& Okon, Y. (2001). Responses of agronomically important crops to inoculation with Azospirillum. Functional Plant Biology, 28(9), 871-879.

30.Bashan, Y., Holguin, G., \& De-Bashan, L. E. (2004). Azospirillum-plant relationships: physiological, molecular, agricultural, and environmental advances (1997-2003). Canadian journal of microbiology, 50(8), 521-577.

31. Pérez-Pazos, J. V., \& Sánchez-López, D. B. (2017). Caracterización y efecto de Azotobacter, Azospirillum y Pseudomonas asociadas a Ipomoea batata del Caribe Colombiano. Revista Colombiana de Biotecnología, 19(2), 35-46.

32. Datta, C., \& Basu, P. S. (2000). Indole acetic acid production by a Rhizobium species from root nodules of a leguminous shrub, Cajanus cajan. Microbiological research, 155(2), 123-127.

33. Mahato, S., \& Kafle, A. (2018). Comparative study of Azotobacter with or without other fertilizers on growth and yield of wheat in Western hills of Nepal. Annals of Agrarian Science, 16(3), 250-256.

34.Aasfar, A., Bargaz, A., Yaakoubi, K., Hilali, A., Bennis, I., Zeroual, Y., \& Meftah Kadmiri, I. (2021). Nitrogen Fixing Azotobacter Species as Potential Soil Biological Enhancers for Crop Nutrition and Yield Stability. Frontiers in Microbiology, 12, 354.

35.Loredo-Osti, C., López-Reyes, L., \& Espinosa-Victoria, D. (2004). Bacterias promotoras del crecimiento vegetal asociadas con gramíneas: Una revisión. Terra Latinoamericana, 22(2), 225-239.

Received: 3 Agosto 2021

Accepted: 10 Octubre 2021 